\title{
PD-1 and PD-L1 correlated gene expression profiles and their association with clinical outcomes of breast cancer
}

\author{
Cui Jiang ${ }^{1}$, SunRun $\mathrm{CaO}^{2}$, Na Li ${ }^{2}$, Lei Jiang ${ }^{1}$ and Tao Sun ${ }^{1 *}$
}

\begin{abstract}
Background: Immunotherapies that targeting programmed cell death 1 (PD-1) and programmed death-ligand 1 (PD-L1) have obtained prominent success in breast cancer (BC). However, not all the patients benefit from the antibody therapy. This study aimed to identify PD-1/PD-L1 correlated genes and pathways as well as investigate their potential as prognostic marker in BC.
\end{abstract}

Materials and methods: By analysing transcriptional data of BC from TCGA, we identified PD-1 and PD-L1 correlated genes by WGCNA analysis and explored the biological process as well as pathways they enriched. Co-expression analysis were performed for PD-1/PD-L1 with immune infiltration and checkpoints. The prognostic value of PD-1 and PD-L1 were also investigated.

Results: PD-1 and PD-L1 expression showed significant difference in different molecular subtypes and stages. PD-1 correlated genes enriched in T cell activation, lymphocyte activation, leukocyte migration while PD-L1 correlated genes demonstrated enrichment including T cell apoptotic process, tolerance induction and cytolysis. Immune infiltration analysis suggested that PD- 1 and PD-L1 were related with Neutrophils $(r=0.65, r=0.48)$ and Fibroblasts $(r=0.59, r=0.47)$. For immune checkpoints analysis, PD-1 was associated with HLA-A $(r=0.804)$ and INPP5D $(r=0.782)$ while PD-L1 correlated with CTLA4 $(r=0.843)$ and CD27 $(r=0.823)$. PD-1 was associated favorable survival of $B C(H R=0.67, P=0.012)$ while PD-L1 did not demonstrate significant association with $B C$ prognosis $(H R=0.85$, $P=0.313)$.

Conclusion: PD-1 and PD-L1 correlated genes participated in biological process including T cell activation, lymphocyte activation, leukocyte migration, T cell apoptotic process, tolerance induction and cytolysis. PD-1/PD-L1 expression also demonstrated relation with immune infiltration and immune checkpoints. High PD-1 expression predicted better survival of breast cancer patients.

Keywords: PD-1, PD-L1, Immune, Breast cancer

\section{Background}

As one of the most frequently occurred malignant tumors, breast cancer $(\mathrm{BC})$ remains the leading cause of cancer-related death for females in many countries [1]. Breast cancer arises from multiple genetic factors, environmental alternations and their complicated

\footnotetext{
*Correspondence: suntao@cancerhosp-In-cmu.com

${ }^{1}$ Department of Medical Oncology, Cancer Hospital of China Medical University, Liaoning Cancer Hospital and \& Institute, 44 Xiaoheyan Road, Dadong District, Shenyang 110042, Liaoning, People's Republic of China Full list of author information is available at the end of the article
}

interactions [2]. According to the status of biomarkers including estrogen receptor (ER), progesterone receptor (PR) and epidermal growth factor receptor 2 (HER2), patients with breast cancer were classified into groups of luminal A, luminal B, HER-2 positive and triple negative [3]. It has been accepted that different groups of $\mathrm{BC}$ patients benefit from corresponding treatment strategy of chemical and hormonal therapy [4].

Although certain therapeutic combinations have been used as standard treatment in clinical management of 
$\mathrm{BC}$, some $\mathrm{BC}$ patients still could not get satisfactory clinical outcomes [5]. The different outcomes of $\mathrm{BC}$ patients indicated that other critical factors also determine the final therapeutic effect such as the immune status of the cells [6]. It is well-accepted that immune escape of tumor cells and aberrant human immune surveillance play essential role in carcinogenesis, progression and metastasis of various types of cancer [7]. As for immune escape of cancer cells, the identification of PD-1 (programmed death 1) and PD-L1 (programmed death-ligand 1) axis was one of the most encouraging finding of cancer therapy in recent years [8]. Serving as an immune checkpoint in tumor microenvironment, the antibodies of PD-1/ PD-L1 has shown prominent effect in a large number of cancer types [9].

Previously, PD-L1 expression has been reported to be associated with worse prognosis of triple negative breast cancer patients, which counteract effect of tumorinfiltrating lymphocytes (TILs) [10]. Another study of HER2 + invasive BC patients indicated a positive correlation of PD-L1 expression and $\mathrm{CD} 8+\mathrm{T}$ cells with favorable clinical outcomes [11]. One research of 1318 BC patients in European suggested that PD-1 positive immune cells in triple negative breast cancer correlated with longer disease-free survival, and tumor-infiltrating lymphocytes (TILs) density was remarkably related with PD-1 and PD-L1 expression in immune cells [12]. Most of the findings implied that medicine concerning PD-1 and PD-L1 immune checkpoint might become novel therapeutic strategies for breast cancer.

Although the critical role of immune checkpoint PD-1 and PD-L1 have been widely reported in a number of malignant tumors, the underlying regulating mechanisms in breast cancer is still unclear. In this study, we performed comprehensive analysis of gene expression profiles related to PD-1 and PD-L1 in breast cancer using transcriptome data from TCGA. The correlation of PD-1 and PD-L1 with other immune biomarkers and immune cells infiltration were revealed. Furthermore, the effect of PD-1 and PD-L1 on clinical outcomes of BC was explored to determine their potential as biomarkers for $\mathrm{BC}$ patients prognosis.

\section{Materials and methods Analyzed datasets}

The RNA sequencing and clinical data of breast cancer patients in TCGA datasets were downloaded from UCSC XENA (https://xena.ucsc.edu/). The level of gene expression was measured as Transcripts per million reads (TPM). Clinical data included the histological type, molecular Type, cancer stage, recurrence event and survival information. The relationship between PD-1/PD-L1 expression and the clinical data were investigated.

\section{Co-expression gene and enrichment analysis}

Weighted correlation network analysis (WGCNA) is an algorithm for finding genetic interactions in a weighted manner. Co-expressed genes obtained by WGCNA analysis will be more accurate. Using WGCNA analysis, we searched the co-expressed genes for PD-1 and PD-L1. As genes with little variation in expression usually represent noise, the most variant genes were filtered for network construction. Gene variabilities were measured by median absolute deviation (MAD). If the interaction gene was more than 200, we used the interaction degree to search the top 200 gene as the interaction genes. Clusterprofiler is a $R$ package for enrichment analysis. Using clusterprofier, we used biological process in the Gene Ontology (GO) to analyze the interacted genes [13]. Since the results of the enrichment analysis contain many similar results, we further concentrated the results of the enrichment analysis.

\section{Relationship between immune factors and PD-1/PD-L1}

A variety of studies have confirmed that immune infiltration and all aspects of the tumor are related. MCP-counter is available $R$ package to estimate the sample immune infiltration. From a gene expression matrix, it produces for each sample an abundance score for CD3+ T cells, CD8+ T cells, cytotoxic lymphocytes, NK cells, B lymphocytes, cells originating from monocytes (monocytic lineage), myeloid dendritic cells, neutrophils, as well as endothelial cells and fibroblasts. Then we used correlation analysis to evaluate the correlation between PD-1/ PD-L1 and immune infiltration. Immunological checkpoints serve as the primary site for detecting immune status, and we also evaluated the relationship between PD-1 and PD-L1 and immune checkpoints.

\section{Statistical analysis}

In this study, statistical analysis was mainly performed by using $\mathrm{R}$ language (https://www.r-project.org/) with several publicly available packages. Rank sum test was used to evaluate the expression difference of PD-1/PD-L1 in different groups. Spearman correlation analysis was used to explore the correlation between PD-1/PD-L1 expression and immune infiltration and immune checkpoints. Survival curve was generated by Kaplan- Meier method based on log-rank test. Other Figures were generated by several $R$ packages, such as pheatmap, circlize, and corrplot. All multiple tests were corrected by the BH method. A probability value $\mathrm{P}<0.05$ was considered to be significant in this study.

\section{Results}

PD-1/PD-L1 expression status in different clinical subgroups

Using TCGA datasets, we analyzed the PD-1/PD-L1 expression in different groups according to the clinical 
data. As shown in Fig. 1a, both PD-1 and PD-L1 expression showed significant difference in the three molecular subtypes $(\mathrm{P}<0.001$ and $\mathrm{P}=0.047$, respectively). Luminal and Basal-like subtype show significant difference in PD-1 and PD-L1 expression. Moreover, the expression of PD-L1 differs among different stages $(P=0.032)$, while PD-1 did not show any difference $(\mathrm{P}=0.536)$. In addition, both PD-1 and PD-L1 expression correlated with the recurrence event of $\mathrm{BC}$ patients $(\mathrm{P}=0.017, \mathrm{P}=0.015$, respectively). We also analyzed the relation of $\mathrm{PD}-1 /$
PD-L1 expression with clinical data including therapy, histological subtype, ER, PR and HER-2 status (Table 1). Finally, both PD-1 and PD-L1 were correlated with ER, $\mathrm{PR}$ and clinical therapy, indicating the probable implication of PD-1/PD-L1 in clinical outcome.

\section{Co-expression analysis of genes associated with PD-1 and PD-L1}

Using WGCNA, we analyzed the co-expression gene associated with PD-1 and PD-L1. The connectivity among

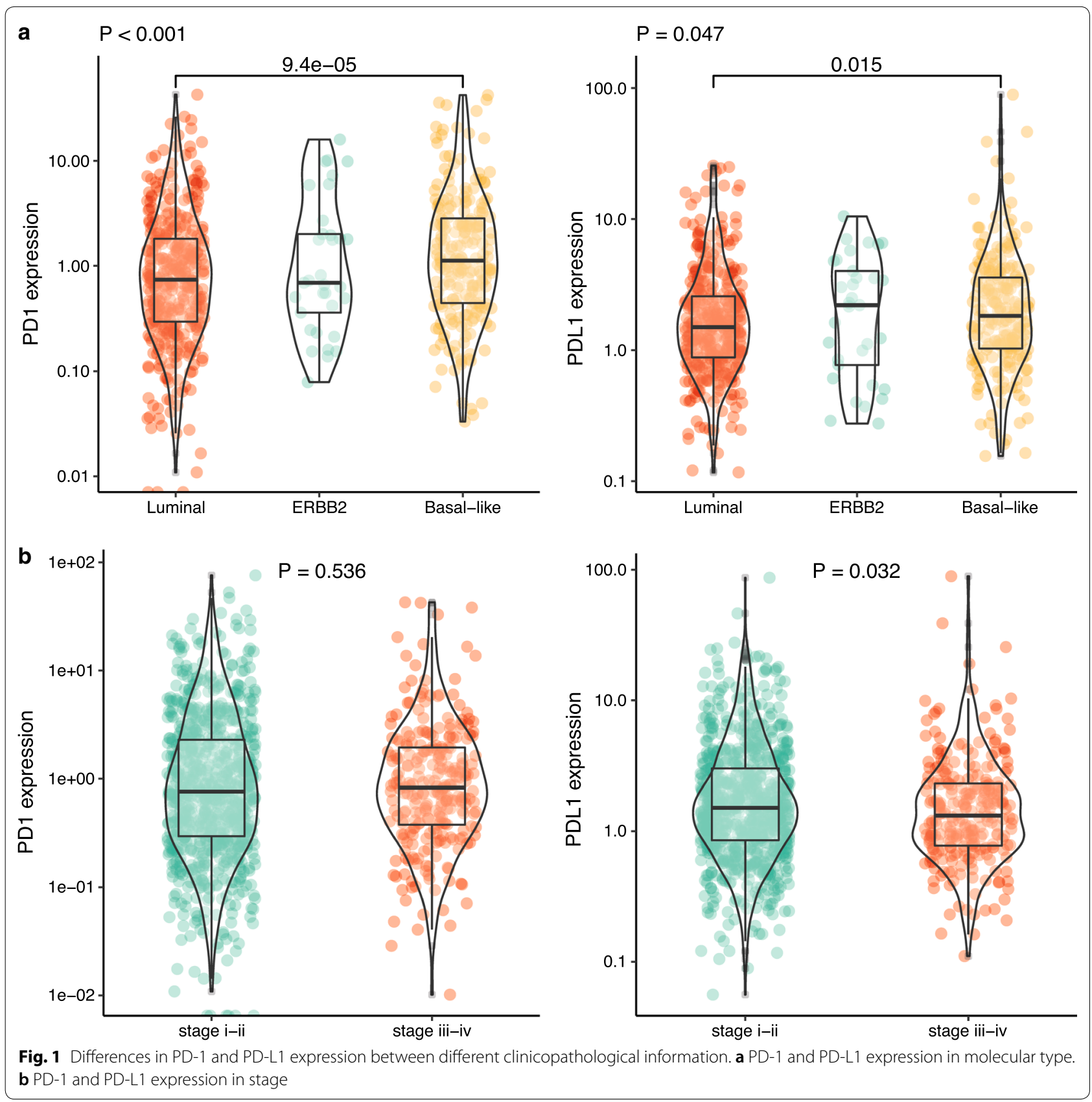


Table 1 Association between PD-1/PD-L1 expression and clinical data

\begin{tabular}{|c|c|c|c|c|c|c|}
\hline Group & Category & $\mathrm{n}$ & PD1 [mean (sd)] & PD1 P & PDL1 [mean (sd)] & $\overline{\text { PDL1 P }}$ \\
\hline ER & Negative & 212 & $4.35(7.21)$ & $<0.001$ & $4.35(7.84)$ & $<0.001$ \\
\hline ER & Positive & 727 & $1.82(3.55)$ & & $2.28(3.19)$ & \\
\hline Her2 & Negative & 495 & $2.54(5.23)$ & 0.27 & $3.04(5.62)$ & 0.39 \\
\hline Her2 & Positive & 152 & $2.04(3.38)$ & & $2.62(4.13)$ & \\
\hline$P R$ & Negative & 308 & $3.47(6.27)$ & $<0.001$ & $3.41(6.43)$ & 0.003 \\
\hline$P R$ & Positive & 628 & $1.86(3.68)$ & & $2.42(3.59)$ & \\
\hline Histological type & Infiltrating ductal carcinoma & 790 & $2.48(5.67)$ & 0.274 & $2.94(5.77)$ & 0.278 \\
\hline Histological type & Infiltrating lobular carcinoma & 205 & $2.58(4.82)$ & & $2.36(2.39)$ & \\
\hline Histological type & Other type & 107 & $1.62(4.44)$ & & $2.42(5.08)$ & \\
\hline Therapy type & Chemotherapy & 385 & $3.14(6.96)$ & 0.015 & $3.38(6.38)$ & 0.004 \\
\hline Therapy type & Hormone therapy & 365 & $1.96(4.24)$ & & $2.18(2.82)$ & \\
\hline Therapy type & Other therapy & 31 & $2.02(2.24)$ & & $2.56(2.19)$ & \\
\hline
\end{tabular}
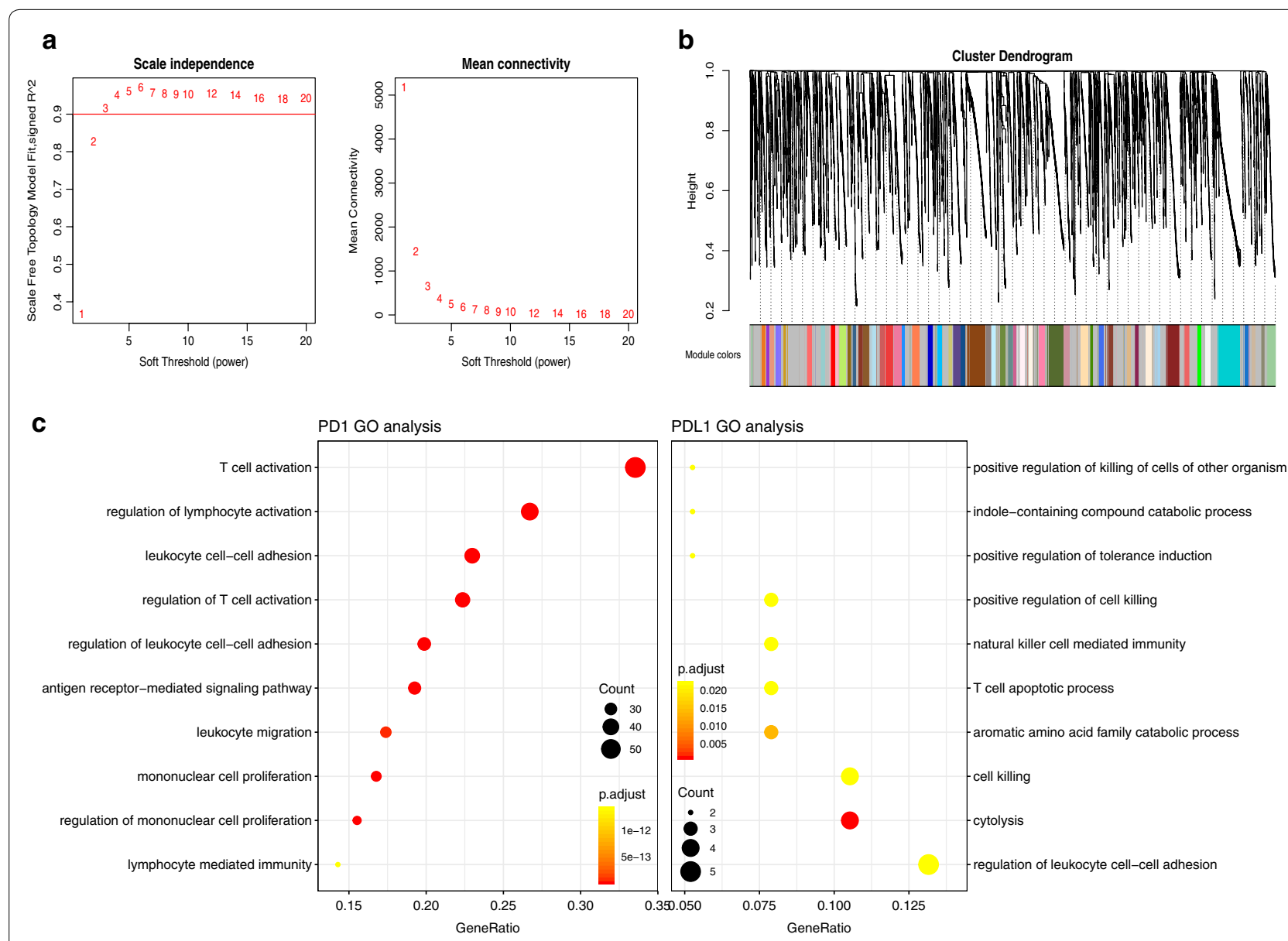

PDL1 GO analysis

Fig. 2 Co-expression analysis of genes associated with PD-1 and PD-L1. a Soft threshold selection in the WGCNA network analysis. b Gene distribution in the WGCNA network analysis. c GO analysis for the PD-1 and PD-L1 co-expression genes

genes was a scale-free network distribution if the value of soft thresholding power $\beta$ equals to 3 (Fig. 2a). Altogether 21 module was obtained according to WGCNA analysis (Fig. 2b). Among these modules, PD-1 belonged to pink module while PD-L1 belonged to thistle 1 module. We finally got 1065 genes that interacted with PD-1 and 99 PD-L1 correlated genes. Then we selected the top 200 gene associated with PD-1 and all of the 99 PD-L1 related 
genes for further enrichment analysis. PD-1 correlated genes mainly enriched in biological process of $\mathrm{T}$ cell activation, regulation of lymphocyte activation, regulation of $\mathrm{T}$ cell activation and leukocyte migration while PD-L1 correlated genes demonstrated enrichment including positive regulation of killing of cells of other organism, $\mathrm{T}$ cell apoptotic process, positive regulation of tolerance induction and cytolysis (Fig. 3 and Table 2).

\section{PD-1/PD-L1 expression and immune infiltration}

Using Microenvironment Cell Populations-counter, we evaluated the profiles of immune infiltration among various subtypes and stages breast cancer (Fig. 3a). Additionally, the associations of PD-1 and PD-L1 with immune cell populations according to the transcriptomic data were analyzed. The results indicated that PD-1 and PD-L1 were mainly related with Neutrophils $(\mathrm{r}=0.65, \mathrm{r}=0.48)$ and Fibroblasts $(\mathrm{r}=0.59, \mathrm{r}=0.47)$ (Fig. 3b).

\section{PD-1/PD-L1 expression and immune checkpoints}

As previous reported, the immune checkpoints mainly included CD28, CD80, CD86, CTLA4, INPP5D, INPPL1, CD58, CD27, CD70, HLA-A, CD74. We then analyzed the

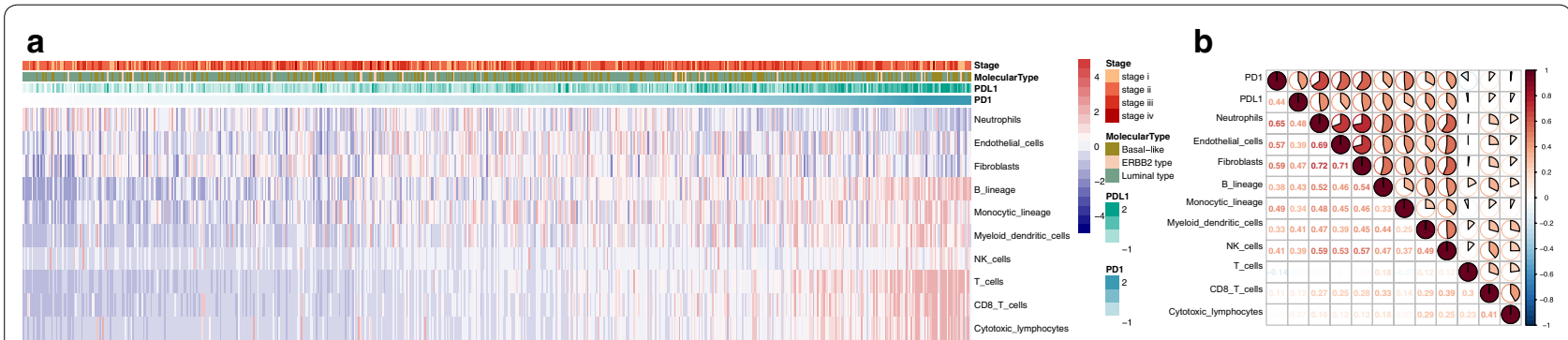

Fig. 3 PD-1/PD-L1 expression and immune infiltration. a The proportion of all immune infiltration components in breast cancer. $\mathbf{b}$ co-expression analysis between PD-1/PD-L1 and immune infiltration

Table 2 Top ten terms of GO analysis for PD-1 and PD-L1

\begin{tabular}{|c|c|c|c|c|c|}
\hline Gene & Description & GENERATIO & $P$ value & $P$ adjust & Count \\
\hline \multirow[t]{12}{*}{ PD1 } & T cell activation & $54 / 161$ & 8.67E-47 & $1.53 \mathrm{E}-43$ & 54 \\
\hline & Regulation of lymphocyte activation & $43 / 161$ & $8.13 E-32$ & $7.17 \mathrm{E}-29$ & 43 \\
\hline & Leukocyte cell-cell adhesion & $37 / 161$ & $9.69 \mathrm{E}-31$ & 4.27E-28 & 37 \\
\hline & Regulation of T cell activation & $36 / 161$ & $1.45 \mathrm{E}-30$ & $5.11 \mathrm{E}-28$ & 36 \\
\hline & Antigen receptor-mediated signaling pathway & $31 / 161$ & $1.61 E-26$ & $4.74 \mathrm{E}-24$ & 31 \\
\hline & Regulation of leukocyte cell-cell adhesion & $32 / 161$ & $4.29 E-26$ & $1.08 \mathrm{E}-23$ & 32 \\
\hline & Regulation of mononuclear cell proliferation & $25 / 161$ & $1.22 \mathrm{E}-21$ & $1.13 \mathrm{E}-19$ & 25 \\
\hline & Mononuclear cell proliferation & $27 / 161$ & $3.08 \mathrm{E}-21$ & $2.59 E-19$ & 27 \\
\hline & Leukocyte migration & $28 / 161$ & $1.13 E-15$ & $6.04 \mathrm{E}-14$ & 28 \\
\hline & Lymphocyte mediated immunity & $23 / 161$ & $3.15 E-14$ & $1.43 \mathrm{E}-12$ & 23 \\
\hline & Adaptive immune response & $22 / 161$ & $5.23 \mathrm{E}-13$ & $2.15 \mathrm{E}-11$ & 22 \\
\hline & Regulation of leukocyte mediated immunity & $16 / 161$ & $1.61 \mathrm{E}-11$ & $5.57 \mathrm{E}-10$ & 16 \\
\hline \multirow[t]{12}{*}{ PDL1 } & Cytolysis & $4 / 38$ & $1.18 \mathrm{E}-06$ & 0.00130442 & 4 \\
\hline & Aromatic amino acid family catabolic process & $3 / 38$ & $2.53 \mathrm{E}-05$ & 0.01394493 & 3 \\
\hline & T cell apoptotic process & $3 / 38$ & 0.00014677 & 0.02200257 & 3 \\
\hline & Positive regulation of tolerance induction & $2 / 38$ & 0.0001831 & 0.02200257 & 2 \\
\hline & Indole-containing compound catabolic process & $2 / 38$ & 0.0001831 & 0.02200257 & 2 \\
\hline & Positive regulation of killing of cells of other organism & $2 / 38$ & 0.00026786 & 0.02200257 & 2 \\
\hline & Natural killer cell mediated immunity & $3 / 38$ & 0.00027843 & 0.02200257 & 3 \\
\hline & Cell killing & $4 / 38$ & 0.00030195 & 0.02200257 & 4 \\
\hline & Positive regulation of cell killing & $3 / 38$ & 0.00030586 & 0.02200257 & 3 \\
\hline & Regulation of leukocyte cell-cell adhesion & $5 / 38$ & 0.00031554 & 0.02200257 & 5 \\
\hline & Regulation of killing of cells of other organism & $2 / 38$ & 0.00036836 & 0.02200257 & 2 \\
\hline & Positive regulation of $\mathrm{T}$ cell apoptotic process & $2 / 38$ & 0.00036836 & 0.02200257 & 2 \\
\hline
\end{tabular}




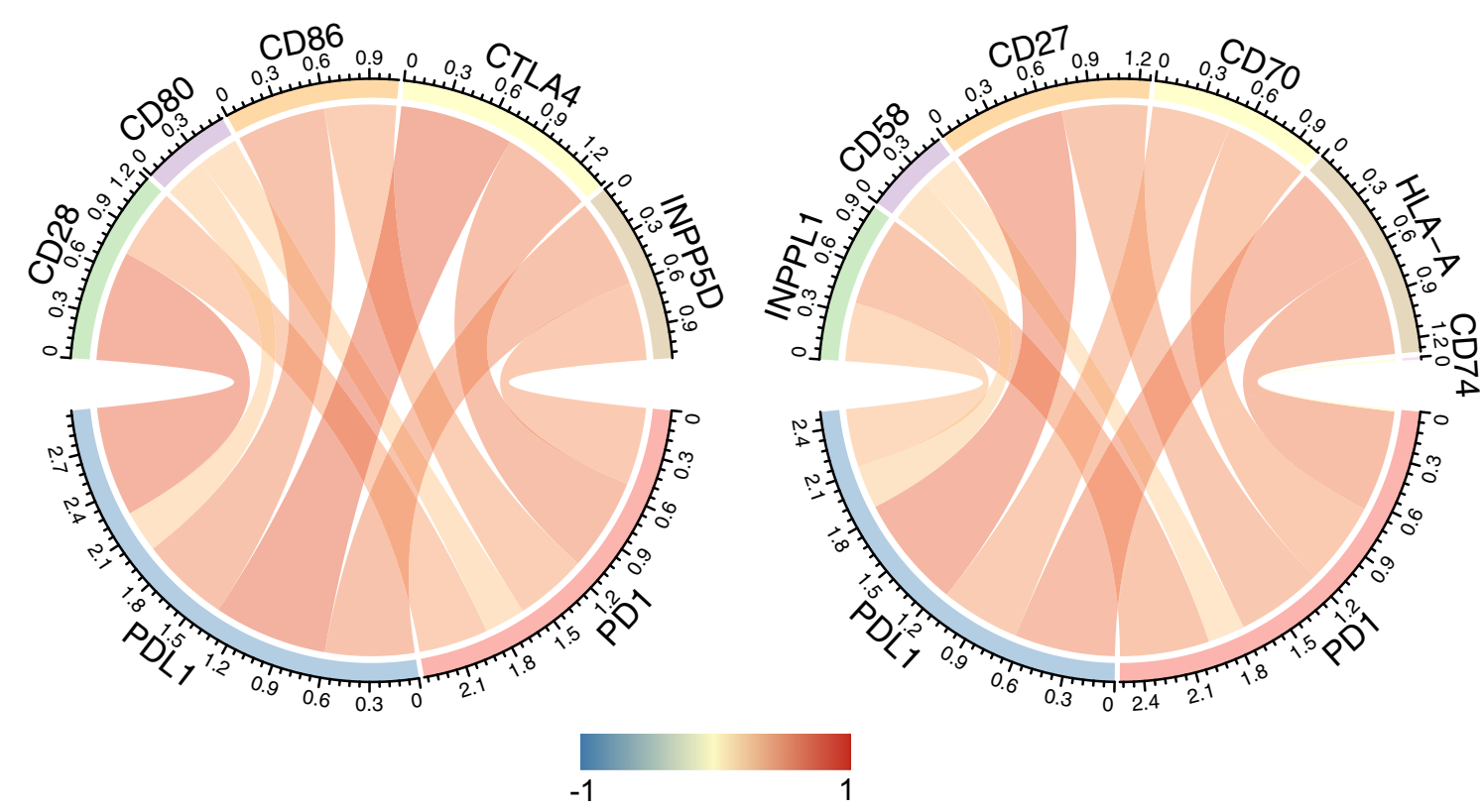

Fig. 4 Co-expression analysis between PD-1/PD-L1 and immune checkpoints

Table 3 Co-expression analysis between PD-1/PD-L1 and immune checkpoints

\begin{tabular}{lllllll}
\hline Gene & PD1_R & PD1_p & PD1_padj & PDL1_R & PDL1_p & PDL1_padj \\
\hline CD28 & 0.68898768 & $3.05 E-156$ & $6.72 E-156$ & 0.72957675 & $4.77 E-184$ & $1.31 E-183$ \\
CD80 & 0.50168845 & $1.98 E-71$ & $2.18 E-71$ & 0.46293539 & $9.94 E-60$ & $1.09 E-59$ \\
CD86 & 0.61700599 & $8.49 E-117$ & $1.17 E-116$ & 0.70665274 & $9.35 E-168$ & $1.71 E-167$ \\
CTLA4 & 0.7430565 & $1.96 E-194$ & $5.39 E-194$ & 0.84321417 & $2.52 E-299$ & $2.77 E-298$ \\
INPP5D & 0.78284443 & $2.58 E-229$ & $1.42 E-228$ & 0.6726487 & $2.64 E-146$ & $4.15 E-146$ \\
INPPL1 & 0.6491299 & $4.48 E-133$ & $7.03 E-133$ & 0.61353869 & $3.75 E-115$ & $5.16 E-115$ \\
CD58 & 0.61591686 & $2.80 E-116$ & $3.43 E-116$ & 0.59168023 & $3.10 E-105$ & $3.79 E-105$ \\
CD27 & 0.74560817 & $1.79 E-196$ & $6.56 E-196$ & 0.82368263 & $9.05 E-274$ & $4.98 E-273$ \\
CD70 & 0.68253159 & $3.08 E-152$ & $5.65 E-152$ & 0.72220769 & $1.24 E-178$ & $2.74 E-178$ \\
HLA-A & 0.80464345 & $9.84 E-252$ & $1.08 E-250$ & 0.74085677 & $1.08 E-192$ & $3.94 E-192$ \\
CD74 & 0.1938974 & $8.19 E-11$ & $8.19 E-11$ & 0.16155305 & $6.77 E-08$ & $6.77 E-08$ \\
\hline PVal & & & & &
\end{tabular}

Pvalue was adjusted by 'BH' methods

correlation between PD-1/PD-L1 expression and important immune checkpoints. As shown in Fig. 4 and Table 3, PD-1 was mainly associated with HLA-A $(r=0.804)$ and INPP5D $(r=0.782)$ while PD-L1 correlated with CTLA4 $(r=0.843)$ and $\mathrm{CD} 27(\mathrm{r}=0.823)$.

\section{Survival analysis of PD-1/PD-L1 and PD-1/PD-L1 correlated genes}

As PD-1/PD-L1 expression was correlated with clinical data, we then explored the prognostic value of PD-1/PD-L1 in breast cancer. As shown in Fig. 5 and Table 4, the expression of PD-1 was associated with favorable survival of breast cancer patients $(\mathrm{HR}=0.67$, 95\% CI0.49-0.91, $\mathrm{P}=0.012$ ) while PD-L1 did not demonstrate significant association with $\mathrm{BC}$ prognosis $(\mathrm{HR}=0.85,95 \% \mathrm{CI} 0.62-1.17, \mathrm{P}=0.313)$. As for the multivariable analysis adjusting for age and stage, PD-1 still predicted better survival for $B C$ patients $(H R=0.71$, 95\% CI0.50-0.99, $\mathrm{P}=0.045)$ but $\mathrm{PD}-\mathrm{L} 1$ showed no significant relation $(\mathrm{HR}=0.99,95 \% \mathrm{CI} 0.71-1.37, \mathrm{P}=0.934)$. In addition, PD-1/PD-L1 correlated genes were also analyzed in relation to prognosis of $\mathrm{BC}$ patients. The results suggested that certain immune markers interacting with PD-1/PD-L1 also correlated with survival of BC patients 

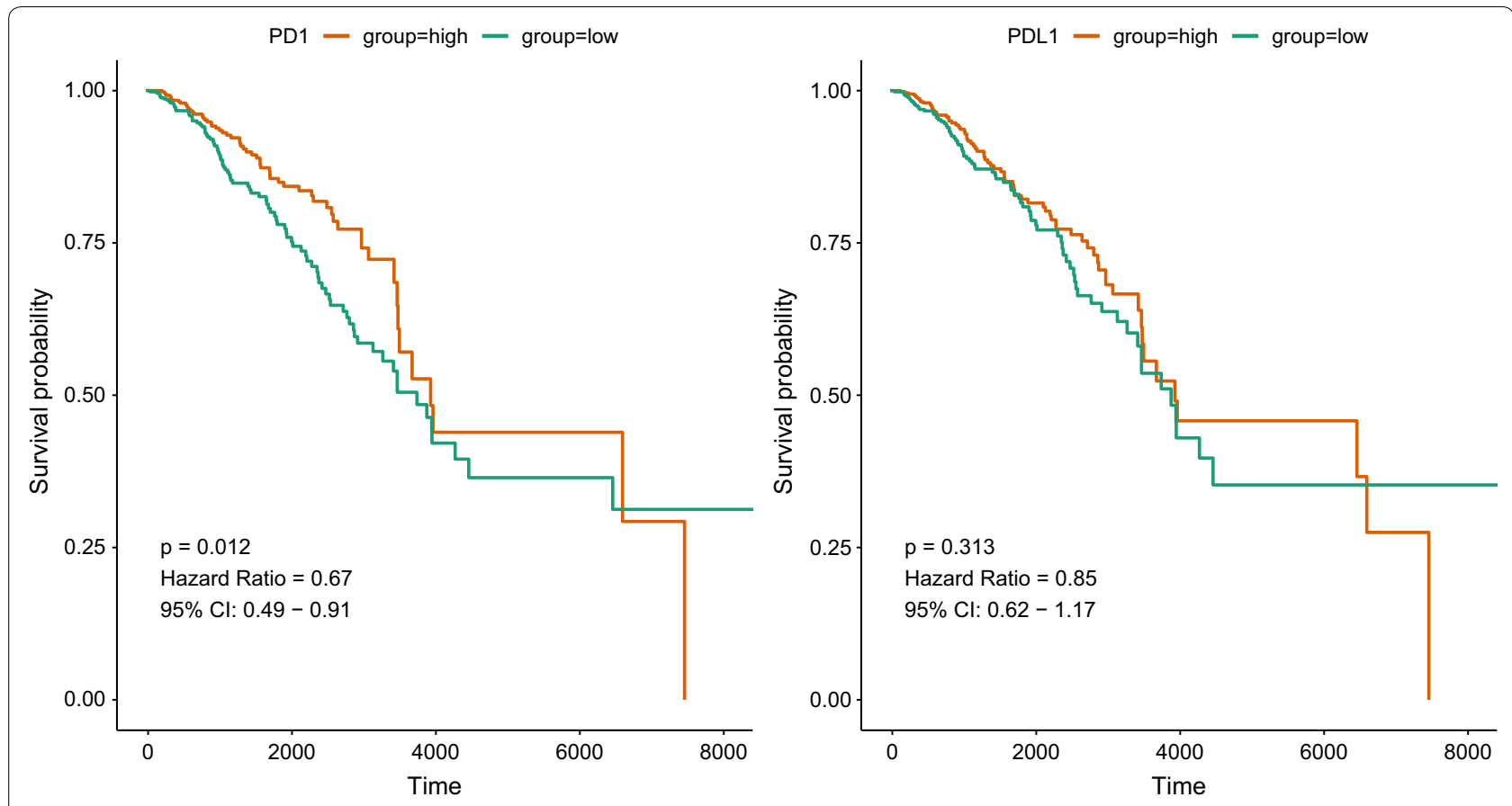

Fig. 5 Prognostic analysis of PD-1/PD-L1

Table 4 Cox regression for PD-1 and PD-L1

\begin{tabular}{lllll}
\hline Gene & HR $(\mathbf{9 5} \% \mathbf{C l})$ & $\mathbf{P}$ & HR $(\mathbf{9 5} \% \mathbf{C l})$ adjusted & P adjusted \\
\hline PD1 & $0.67(0.49-0.91)$ & 0.012 & $0.71(0.50-0.99)$ & 0.045 \\
PDL1 & $0.85(0.62-1.17)$ & 0.313 & $0.99(0.71-1.37)$ & 0.934 \\
\hline
\end{tabular}

Adjust by stage and age

including CD5, CD74, CD96 and CD226 (Additional file 1: Table S1).

\section{Discussion}

It is well-accepted that tumor and its microenvironment is a complex unit to complete the aggressive growth and metastasis of cancer cells [14-16]. Although a number of chemical and radical therapy have been used in clinical practice of cancer treatment, the immune system of human are still believed to be the most fundamental and effective weapon against cancer [17]. Programmed cell death 1 (PD-1) and corresponding ligand (PD-L1) are one of the most critical biological suppressors of cytotoxic immune reaction, the antibody of which has been authorized by FDA in a unprecedented fast term $[18,19]$. However, the inhibitor of PD-1/PD-L1 were not effective in every individual, which require the comprehensive understanding of specific mechanisms underlying PD-1/ PD-L1 regulation in carcinogenesis [20].
Using data from TCGA, we first unraveled the expression status of PD-1/PD-L1 in different subtypes and clinical stages of BC patients. PD-L1 expression was decreased in stage III-IV compared with stage I-II. On the basis of molecular classification, basal-like $\mathrm{BC}$ subtype showed highest expression of PD-1 and ERBB2 subtype $\mathrm{BC}$ had highest PD-L1 expression, which suggest that different subtypes possess various PD-1/PD-L1 status. In addition, PD-1 and PD-L1 expression correlated with the recurrence of $\mathrm{BC}$ patients, which also confirm the critical role of PD-1/PD-L1 immune checkpoint in $\mathrm{BC}$ progression.

We subsequently identified co-expressed genes of PD-1 and PD-L1 by means of WGCNA. A total of 1065 genes correlated with PD-1 and 99 PD-L1 correlated genes were screened. The GO enrichment analysis of PD-1 correlated genes suggested biological process of $\mathrm{T}$ cell activation, regulation of lymphocyte activation, regulation of $\mathrm{T}$ cell activation and leukocyte migration. In addition, PD-L1 correlated genes demonstrated enrichment including positive regulation of killing of cells of other organism, $\mathrm{T}$ cell apoptotic process, positive regulation of tolerance induction and cytolysis. The results of previous studies were in accordance with the GO enrichment of PD-1/PD-L1 related genes in functional modulation of $\mathrm{T}$ cell. For instance, one study suggested that during the transition from DCIS to an invasive lesion, the host cytolytic $\mathrm{T}$ cells interacted with the tumor and destroy 
the tumor tissue, leading to an adaptive upregulation of PD-L1 and tumor protection against immune destruction [21]. PD-L1 is expressed by antigen-presenting cells and results in $\mathrm{T}$ cell inactivation by interaction with $\mathrm{PD}-1$ on T-cells. It is therefore of great importance to clarify the biological process and pathways we identified for PD-1/ PDL1 related genes, by which immunotherapy with PD-1- and PD-L1-targeted monoclonal antibodies might dramatically change the therapeutic and prognostic landscape for cancer.

Immune infiltration of breast cancer determine the immune activation of tumor microenvironment and is related with clinical outcome of patients. According to the correlation analysis of PD-1 and PD-L1 with immune cell populations, PD-1 and PD-L1 were mainly related with Neutrophils and Fibroblasts. It has been reported that PD-1 protein expression significantly correlated with higher TIL abundance, Ki-67 index, basallike subtypes, and distant metastasis of triple-negative breast cancer (TNBC) [22]. The interaction of PD-1/ PD-L1 immune checkpoint with immune infiltration is a promising research direction in the future. The immune checkpoints of CD28, CD80, CD86, CTLA4, INPP5D, INPPL1, CD58, CD27, CD70, HLA-A, CD74 were also analyzed in relation to PD-1/PD-L1 expression. Finally, PD-1 was found to be mainly associated with HLA-A and INPP5D while PD-L1 correlated with CTLA4 and CD27. Cytotoxic T-lymphocyte-associated protein 4 (CTLA4) belongs to immunoglobulin superfamily and encodes a protein transmitting inhibitory signal to $\mathrm{T}$ cells, the antibody of which also demonstrated favorable outcome in clinical management [23]. The combination usage of PD-1/PD-L1 with these immune checkpoints might become novel therapeutic targets in the future [24].

Survival analysis of PD-1/PD-L1 expression status demonstrated that the expression of PD-1 was associated favorable survival of breast cancer patients while PD-L1 did not suggest significant association with BC prognosis. In a study of 195 triple-negative breast cancer individuals, PD-1 was found to be significantly related with better disease free survival and overall survival. The results of this study also suggested that PD-1 protein expression in TILs, but not PD-L1 in tumor cells, predicted better prognosis in TNBC [22]. Researchers demonstrated that high expression of PDL1 are associated with favorable clinical outcome in 127 primary breast cancer [25]. Another study of HER2+ invasive BC patients indicated a positive correlation of PD-L1 expression and $\mathrm{CD} 8+\mathrm{T}$ cells with favorable clinical outcomes [11]. On contrary, PD-L1 expression has been reported to be associated with worse prognosis of triple negative breast cancer patients, which counteract effect of tumorinfiltrating lymphocytes [10]. Response of BC patients to neoadjuvant therapy and survival outcome indicated that PD-L1 predicted better rate of pathological complete response (pCR) [26]. In addition, PD-1/PD-L1 correlated genes such as CD5, CD74, CD96 and CD226 were also related with prognosis of $\mathrm{BC}$ patients. The combination of these immune markers with PD-1/PD-L1 might improve the prediction and management of $\mathrm{BC}$ patients in the future. Until now, whether PD-1/PL-L1 predict prognosis in $\mathrm{BC}$ patients was still in debate. The specific association of PD-1/PD-L1 with BC prognosis still required further large-scale investigations to confirm, which would benefit the potential of PD-1/PD-L1 as prognostic biomarker.

\section{Conclusion}

In this study, we reported the expression status of PD-1 and PD-L1 in different subtypes of breast cancer. The PD-1/PDL1 correlated gene profiles were described, the enrichment analysis of which focus on biological process including $\mathrm{T}$ cell activation, lymphocyte activation, leukocyte migration, $\mathrm{T}$ cell apoptotic process, tolerance induction and cytolysis. PD-1/PD-L1 expression also demonstrated relation with immune cells infiltration and multiple immune checkpoints. High PD-1 expression was associated with better survival of breast cancer patients.

\section{Supplementary information}

Supplementary information accompanies this paper at https://doi. org/10.1186/s12935-019-0955-2.

Additional file 1: Table S1. Prognostic analysis of PD1/PDL1 co-expressing genes.

\section{Abbreviations}

PD-1: programmed cell death 1; PD-L1: programmed death-ligand 1; BC: breast cancer; ER: estrogen receptor; PR: progesterone receptor; HER2: epidermal growth factor receptor 2; TILs: tumor-infiltrating lymphocytes; TPM: transcripts per million reads; WGCNA: weighted correlation network analysis; MAD: median absolute deviation; GO: Gene Ontology; Monocytic lineage: cells originating from monocytes; TNBC: triple-negative breast cancer; CTLA4: cytotoxic T-lymphocyte-associated protein 4; pCR: pathological complete response.

\section{Acknowledgements \\ Not applicable.}

\section{Authors' contributions}

$\mathrm{CJ}$ : analyze the data and write the paper. SC: repeated data analysis. NL: reviewed the manuscript. LJ: made the contribution to the data collection. TS: designed the work and reviewed the manuscript. All authors read and approved the final manuscript.

\section{Funding}

This work was supported by the Liaoning Province Key Laboratory Project of Breast Cancer Research [Grant Number 2016-26-1]; Key Research Project of Liaoning [Grant Number 2018225076]; Special Fund for Clinical Research of Wu Jieping Medical Foundation [Grant Number 320.6750.18541] and CSCOHengrui Cancer Research Foundation [Grant Number Y-HR2018-362]. 


\section{Availability of data and materials}

The data that support the findings of this study are available from TCGA.

\section{Ethics approval and consent to participate}

Not applicable.

\section{Consent for publication}

Not applicable.

\section{Competing interests}

The authors declare that they have no competing interests.

\section{Author details}

1 Department of Medical Oncology, Cancer Hospital of China Medical University, Liaoning Cancer Hospital and \& Institute, 44 Xiaoheyan Road, Dadong District, Shenyang 110042, Liaoning, People's Republic of China. ${ }^{2}$ Institute of Translational Medicine, China Medical University, No.77 Puhe Road, Shenyang 110001, Liaoning, People's Republic of China.

Received: 30 June 2019 Accepted: 2 September 2019

Published online: 09 September 2019

\section{References}

1. Duijf PHG, Nanayakkara D, Nones K, Srihari S, Kalimutho M, Khanna KK Mechanisms of genomic instability in breast cancer. Trends Mol Med. 2019;25(7):595-611.

2. Venema CM, Bense RD, Steenbruggen TG, Nienhuis HH, Qiu SQ, van Kruchten M, Brown M, Tamimi RM, Hospers GAP, Schroder CP, et al. Consideration of breast cancer subtype in targeting the androgen receptor. Pharmacol Ther. 2019;200:135-47.

3. Yamanouchi K, Kuba S, Eguchi S. Hormone receptor, human epidermal growth factor receptor-2, and $\mathrm{Ki}-67$ status in primary breast cancer and corresponding recurrences or synchronous axillary lymph node metastases. Surg Today. 2019. https://doi.org/10.1007/s00595-019-01831-8.

4. Rani A, Stebbing J, Giamas G, Murphy J. Endocrine resistance in hormone receptor positive breast cancer-from mechanism to therapy. Front Endocrinol. 2019;10:245

5. Ji X, Lu Y, Tian H, Meng X, Wei M, Cho WC. Chemoresistance mechanisms of breast cancer and their countermeasures. Biomed Pharmacother 2019:114:108800.

6. Pernas S, Tolaney SM. HER2-positive breast cancer: new therapeutic frontiers and overcoming resistance. Ther Adv Med Oncol. 2019;11:1758835919833519.

7. Ghahremanloo A, Soltani A, Modaresi SMS, Hashemy SI. Recent advances in the clinical development of immune checkpoint blockade therapy. Cell Oncol (Dordrecht). 2019. https://doi.org/10.1007/s13402-019-00456-w.

8. Yasunaga M. Antibody Therapeutics and Immunoregulation in Cancer and Autoimmune Disease. Semin Cancer Biol. 2019. https://doi.org/10.1016/j. semcancer.2019.06.001.

9. Havel JJ, Chowell D, Chan TA. The evolving landscape of biomarkers for checkpoint inhibitor immunotherapy. Nat Rev Cancer. 2019;19(3):133-50.

10. Zhu X, Zhang Q, Wang D, Liu C, Han B, Yang JM. Expression of PD-L1 attenuates the positive impacts of high-level tumor-infiltrating lymphocytes on prognosis of triple-negative breast cancer. Cancer Biol Ther. 2019;20(8):1105-12.
11. Hou Y, Nitta H, Wei L, Banks PM, Lustberg M, Wesolowski R, Ramaswamy B, Parwani AV, Li Z. PD-L1 expression and CD8-positive T cells are associated with favorable survival in HER2-positive invasive breast cancer. Breast J. 2018;24(6):911-9.

12. Noske A, Mobus V, Weber K, Schmatloch S, Weichert W, Kohne CH, Solbach C, Heppner B, Steiger K, Muller V, et al. Relevance of tumour-infiltrating lymphocytes, PD-1 and PD-L1 in patients with high-risk, nodal-metastasised breast cancer of the German Adjuvant Intergroup Node-positive study. Eur J Cancer (Oxford, England: 1990). 2019:114:76-88.

13. Ontology CG. The gene ontology (GO) project in 2006. Nucleic Acids Res. 2006:34(Database issue):D322-6.

14. Garnier L, Gkountidi AO, Hugues S. Tumor-associated lymphatic vessel features and immunomodulatory functions. Front Immunol. 2019;10:720.

15. Singh S, Chakrabarti R. Consequences of EMT-driven changes in the immune microenvironment of breast cancer and therapeutic response of cancer cells. J Clin Med. 2019:8(5):642.

16. Wu L, Saxena S, Awaji M, Singh RK. Tumor-associated neutrophils in cancer: going pro. Cancers. 2019;11(4):564.

17. Longo V, Brunetti O, Azzariti A, Galetta D, Nardulli P, Leonetti F, Silvestris N. Strategies to improve cancer immune checkpoint inhibitors efficacy, other than abscopal effect: a systematic review. Cancers. 2019;11(4):539.

18. Force J, Leal JHS, McArthur HL. Checkpoint blockade strategies in the treatment of breast cancer: where we are and where we are heading. Curr Treat Options Oncol. 2019;20(4):35.

19. O'Donnell JS, Long GV, Scolyer RA, Teng MW, Smyth MJ. Resistance to PD1/ PDL1 checkpoint inhibition. Cancer Treat Rev. 2017;52:71-81.

20. Constantinidou A, Alifieris C, Trafalis DT. Targeting programmed cell death -1 (PD-1) and ligand (PD-L1): a new era in cancer active immunotherapy. Pharmacol Ther. 2019;194:84-106.

21. Lv S, Wang S, Qiao G, Wang X, Zhou X, Yan F, Li Y, Wang S, Morse MA, Hobeika A, et al. Functional CD3(+)CD8(+)PD1(-) T cell accumulation and PD-L1 expression increases during tumor invasion in DCIS of the breast. Clin Breast Cancer. 2019. https://doi.org/10.1016/j.clbc.2019.04.001.

22. Ren $X, W u$ H, Lu J, Zhang Y, Luo Y, Xu Q, Shen S, Liang Z. PD1 protein expression in tumor infiltrated lymphocytes rather than PDL1 in tumor cells predicts survival in triple-negative breast cancer. Cancer Biol Ther. 2018;19(5):373-80

23. Ha D, Tanaka A, Kibayashi T, Tanemura A, Sugiyama D, Wing JB, Lim EL, Teng KWW, Adeegbe D, Newell EW, et al. Differential control of human Treg and effector T cells in tumor immunity by Fc-engineered anti-CTLA-4 antibody. Proc Natl Acad Sci USA. 2019;116(2):609-18.

24. Rotte A Combination of CTLA-4 and PD-1 blockers for treatment of cancer. J Exp Clin Cancer Res. 2019;38(1):255.

25. Uhercik M, Sanders AJ, Owen S, Davies EL, Sharma AK, Jiang WG, Mokbel K. Clinical significance of PD1 and PDL1 in human breast cancer. Anticancer Res. 2017;37(8):4249-54

26. Wu Z, Zhang L, Peng J, Xu S, Zhou L, Lin Y, Wang Y, Lu J, Yin W, Lu J. Predictive and prognostic value of PDL1 protein expression in breast cancer patients in neoadjuvant setting. Cancer Biol Ther. 2019;20(6):941-7.

\section{Publisher's Note}

Springer Nature remains neutral with regard to jurisdictional claims in published maps and institutional affiliations.

Ready to submit your research? Choose BMC and benefit from

- fast, convenient online submission

- thorough peer review by experienced researchers in your field

- rapid publication on acceptance

- support for research data, including large and complex data types

- gold Open Access which fosters wider collaboration and increased citations

- maximum visibility for your research: over 100M website views per year

At BMC, research is always in progress.

Learn more biomedcentral.com/submissions 